\title{
Interdroplet bilayer arrays in millifluidic droplet traps from 3D-printed moulds $\dagger$
}

\author{
Philip H. King, Gareth Jones, Hywel Morgan, Maurits R. R. de Planque \\ and Klaus-Peter Zauner*
}

In droplet microfluidics, aqueous droplets are typically separated by an oil phase to ensure containment of molecules in individual droplets of nano-to-picoliter volume. An interesting variation of this method involves bringing two phospholipid-coated droplets into contact to form a lipid bilayer in-between the droplets. These interdroplet bilayers, created by manual pipetting of microliter droplets, have proved advantageous for the study of membrane transport phenomena, including ion channel electrophysiology. In this study, we adapted the droplet microfluidics methodology to achieve automated formation of interdroplet lipid bilayer arrays. We developed a 'millifluidic' chip for microliter droplet generation and droplet packing, which is cast from a 3D-printed mould. Droplets of 0.7-6.0 $\mu \mathrm{L}$ volume were packed as homogeneous or heterogeneous linear arrays of 2-9 droplets that were stable for at least six hours. The interdroplet bilayers had an area of up to $0.56 \mathrm{~mm}^{2}$, or an equivalent diameter of up to $850 \mu \mathrm{m}$, as determined from capacitance measurements. We observed osmotic water transfer over the bilayers as well as sequential bilayer lysis by the pore-forming toxin melittin. These millifluidic interdroplet bilayer arrays

Received 20th September 2013, Accepted 2nd December 2013

DOI: $10.1039 / c 31 c 51072 \mathrm{~g}$

www.rsc.org/loc combine the ease of electrical and optical access of manually pipetted microdroplets with the automation and reproducibility of microfluidic technologies. Moreover, the 3D-printing based fabrication strategy enables the rapid implementation of alternative channel geometries, e.g. branched arrays, with a design-to-device time of just 24-48 hours.

\section{Introduction}

In droplet microfluidics, aqueous droplets are separated by a bulk oil phase that typically contains surfactant molecules to stabilize the aqueous-oil interface, preventing coalescence when two droplets come in close proximity. ${ }^{1-3}$ With phospholipid molecules as surfactants, droplet-droplet contact leads to the expulsion of the interdroplet oil film and the formation of an interdroplet lipid bilayer, ${ }^{4,5}$ as illustrated in Fig. 1. In recent years this variation on the droplet microfluidics methodology has gained considerable interest because of its potential for the study of bilayer-incorporated membrane proteins ${ }^{6}$ and the creation of droplet networks with collective properties that originate from ion channel or nanopore-mediated transfer of (bio)chemical species over the lipid bilayer. ${ }^{7}$

\section{Interdroplet bilayer arrays}

To date, lipid bilayer-connected droplet networks have been constructed with two quite different methods: manual

Electronics and Computer Science \& Institute for Life Sciences, University of Southampton, Southampton, SO17 1BJ, UK. E-mail: kpz@ecs.soton.ac.uk

$\dagger$ Electronic supplementary information (ESI) available. See DOI: 10.1039/ c3lc51072g pipetting of a small number of microliter droplets into a bulk oil reservoir, ${ }^{4,8}$ or microfluidic generation and positioning of nanoliter-to-picoliter droplets in microfluidic channels or chambers. $^{9-11}$ Microliter droplets-in-oil are large enough for wire electrodes to be inserted. Two droplets can subsequently be contacted by moving their electrodes towards each other, ${ }^{7,8}$ or by electrokinetic manipulation on a planar microelectrode array. ${ }^{12-14}$ After formation of a single interdroplet bilayer in this fashion, the inserted electrodes enable electrophysiological characterization of bilayer-incorporated ion

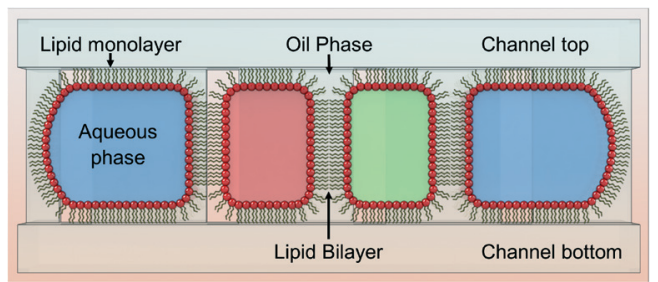

Fig. 1 A droplet-in-oil array contained within a channel. The aqueous droplets are vertically confined by the top and bottom of the channel, and laterally by the channel side walls and by adjacent droplets. Lipid molecules from the oil phase assemble at the water-oil interface as a lipid monolayer. Droplet-droplet contact results in the expulsion of the oil film between the droplets and the formation of an interdroplet lipid bilayer. 
channels or nanopores, which is currently by far the most common application of interdroplet bilayers. ${ }^{4,15}$

Electrically addressable droplets-in-oil have also been used to create droplet networks with collective properties. In a proof of concept study, Bayley and co-workers demonstrated that small networks of two to four interdroplet bilayers with diode-like nanopores are capable of processing electrical input signals, acting as a current limiter or a rectifier. Larger networks can be expected to display more complex collective properties. ${ }^{7}$ Networks of droplets filled with active reaction media are also being explored for molecular information processing. ${ }^{16}$ However, manually pipetting such networks with exact topology and reproducible dimensions is difficult.

In contrast, microfluidic droplet generators produce droplets of a constant volume with droplet packing being determined by the geometry of the microfluidic device. ${ }^{17}$ Given that microfluidic channels are typically 50-500 $\mu \mathrm{m}$ in width and $\approx 50 \mu \mathrm{m}$ in depth, droplet generators tend to be tuned for droplets of 10-100 $\mathrm{nL}$ volume. When the droplet diameter is approximately equal to the channel cross-sectional area, the droplets will pack as a linear array, ${ }^{18}$ and as a $1.5 \mathrm{D}$ array when the channel width is larger. ${ }^{9,11}$ Droplet positioning in microfluidic devices can be guided by intra-channel structural elements, for example semi-circular droplet traps ${ }^{10}$ or pillar arrays, ${ }^{19}$ that enable a static droplet position while maintaining the oil flow through the device. The microfluidic droplet networks reported to date were aimed at bilayer permeability assessment or cross-bilayer communication of chemical oscillators, and employed relatively small interdroplet bilayers in linear network topologies. ${ }^{9-12,18,20,21}$ It should be noted, however, that some groups used a detergent-in-oil rather than a phospholipid-in-oil phase, and hence created interdroplet detergent bilayers, which are not expected to support membrane protein function. ${ }^{10,18}$

\section{Fabrication strategies for microfluidic interdroplet bilayers}

The interdroplet lipid bilayer networks of DeMello and co-workers were produced by inserting tubing, connected to the inlet of a glass capillary or Y-shaped channel, alternately in a bulk water and a bulk oil reservoir with an autosampler, resulting in interdroplet bilayers with a tunable volume between 5 and $100 \mathrm{~nL}$, formed in capillaries and channels of up to $\approx 200$ and $\approx 400 \mu \mathrm{m}$ diameter, respectively. ${ }^{9,11}$ Schmidt and co-workers implemented a pair of classical microfluidic T-junction droplet generators which produced plug-shaped (i.e. channel-confined) droplets of $\approx 5 \mathrm{~nL}$ volume, which were packed in an upstream pillar-flanked microfluidic channel, giving interdroplet lipid bilayers with an estimated maximum diameter of $50 \mu \mathrm{m} .^{20}$ The channels in their droplet array chip were defined by deep reactive ion etching of quartz. The arrays of detergent bilayers $(\approx 10-20 \mu \mathrm{m}$ diameter $)$ mentioned above were also created with on-chip droplet generators, and the microfluidic channels were defined by photolithographic patterning of $\approx 50 \mu \mathrm{m}$ thick photoresist, which served as an inverse mould for feature replication by soft lithography in the transparent elastomer poly(dimethylsiloxane) (PDMS). ${ }^{10,18}$

In terms of mould fabrication for soft lithography, 3D printing (3DP) has recently been proposed as an alternative to lithographic patterning. ${ }^{22,23}$ The minimum 3DP feature size of $\approx 80 \mu \mathrm{m}$ is considerably larger than for photolithography but when high resolution is not required, this disadvantage is offset by the flexibility and the rapid turnaround time of the 3DP technique. Using photolithography, structures higher than $\approx 50-100 \mu \mathrm{m}$ require multiple layers of photoresist to be deposited and baked before patterning and development, whereas layering of millimetres to tens of centimetres or more of 3DP material occurs in a continuous fashion in a single automated process step. 2.5D shapes such as dropletanchoring rails can readily be fabricated by $3 \mathrm{DP},{ }^{24}$ whereas this would require multiple lithography cycles, each with a different photomask. Because the $3 \mathrm{D}$ printer takes its input from 3D computer-aided design (CAD) software, and hence does not depend on masks, which are usually ordered from specialized companies, the design-to-mould time can be less than 24 hours.

Here, we describe microfluidic arrays of densely packed microliter droplets with interdroplet phospholipid bilayers with an area of up to $\approx 0.56 \mathrm{~mm}^{2}$ (equivalent to $850 \mu \mathrm{m}$ diameter). These droplet networks combine the wellcharacterized properties of large interdroplet bilayers with the reproducibility of microfluidic droplet generation and droplet packing. The interdroplet lipid bilayers are characterized by electrical measurements while the action of the membrane-perturbing peptide melittin, as well as the effect of a cross-bilayer salt gradient, is visualized with a camera. The developed millifluidic PDMS chip consists of channels with 2.5D elements for droplet guidance, cast from a 3D-printed mould. Following optimization of fabrication procedures, it was possible to produce complete microdroplet devices with a revised channel layout, including the modification of the CAD file and all the fabrication steps, in about 24-48 hours, which highlights the potential of 3DP for rapid prototyping of 'millifluidic' devices.

\section{Materials and methods}

\section{D-printed mould preparation}

A 3D CAD model of the mould structure was constructed with SolidWorks 2011 (Dassault Systemes, MA, USA), exported as an STL-format file, and printed on a Connex350 ${ }^{\mathrm{TM}} 3 \mathrm{D}$ printing system (Stratasys, MN, USA). The materials used for the mould were either VeroWhitePlus ${ }^{\mathrm{TM}}$, VeroGrey ${ }^{\mathrm{TM}}$ or VeroClear $^{\mathrm{TM}}$, deposited in 'glossy' printer mode on a bed of FullCure®705 support material. The printer slices the structure described in the STL file horizontally. For each slice prepolymer is deposited by the print head, flattened by a roller, and then cured by UV-exposure. Once all layers have been formed, the mould printing is completed. Subsequently, the support material was removed from its underside using a 
high-pressure water jet, and the structure was dried with a stream of compressed air.

The resulting 3D-printed structure is not directly suitable as a mould for PDMS casting because the 3DP material inhibits PDMS polymerization and because the printed structure tends to warp to some extent when it is removed from the printer. We overcame these issues by baking the mould overnight at $80{ }^{\circ} \mathrm{C}$. For this bake step the printed structure was attached to a glass slide by adding a drop of degassed non-polymerized PDMS onto the glass slide, and placing the mould on top, with its base in contact with the PDMS film. The baking temperature is well above the glass transition temperature of $55{ }^{\circ} \mathrm{C}$ of the printed material and allows the heat-relaxed base of the mould $(\approx 2 \mathrm{~mm}$ thick) to flatten out and conform to the surface of the glass. After this procedure, which was successfully applied to VeroWhitePlus ${ }^{\mathrm{TM}}$, VeroGrey $^{\mathrm{TM}}$ and VeroClear ${ }^{\mathrm{TM}}$, the moulds were flat and no longer inhibited the curing of PDMS (see ESI $\dagger$ ). The glass-backed mould was then coated with a layer of trichloro( $1 H, 1 H, 2 H, 2 H$-perfluorooctyl)silane (Sigma Aldrich, MO, USA) by exposure to silane vapour for $1 \mathrm{~h}$ in a vacuum desiccator. An example of a finished mould of VeroGrey ${ }^{\mathrm{TM}}$ material, with outer dimensions of $70 \times 50 \mathrm{~mm}$, is shown in Fig. 2b. The printed mould was cut to obtain a cross-section for optical microscopy. The width and height of the features were larger than the CAD design values (see Fig. 2c), which is expected for 3D printing at this scale with a 600 dpi polymer jet printer (accuracy $\approx 100-200 \mu \mathrm{m}$ ). The CAD design channel width was found to be realised at $\approx 75 \%$ of the measured height of the channels, with respect to the base of the mould, with the material above and below this point being rounded as depicted in Fig. 2c.

\section{PDMS casting and bonding}

Liquid PDMS and curing agent (Sylgard 184 from Dow Corning, MI, USA) were mixed at a 10:1 ratio (w/w) and the mixture was degassed in a vacuum desiccator. The glassbacked mould was then covered with $\approx 4 \mathrm{~mm}$ of liquid PDMS, which was again degassed and then baked at $80{ }^{\circ} \mathrm{C}$ for $1 \mathrm{~h}$. After the polymerized PDMS was detached from the mould, access holes were punched for the fluids $(1.5 \mathrm{~mm}$ diameter) and, if required, for the electrodes ( $1 \mathrm{~mm}$ diameter). Key for a good seal between PDMS and tubing or electrodes is a sharp puncher, we therefore use disposable punches (Kai Medical Disposable Biopsy Punches, Hillside Medical, UK). The top of the PDMS layer was then bonded to a $76 \times 52 \mathrm{~mm}$ glass slide to seal the fluid channels using oxygen plasma treatment (Femto Asher, Diener, Germany, $20 \mathrm{~s}$ at $35-40 \mathrm{~W}$ ). The chip was then baked overnight at $80{ }^{\circ} \mathrm{C}$. This results in improved bonding and also recovers the original hydrophobic PDMS surface chemistry. Finally, the channels of the chip were filled with a $2 \%(\mathrm{v} / \mathrm{v})$ solution of trichloro( $1 H, 1 H, 2 H, 2 H$-perfluorooctyl)silane in ethanol. Following a $30 \mathrm{~min}$ incubation, the channels were flushed with ethanol and the chip was gently dried with a nitrogen gas
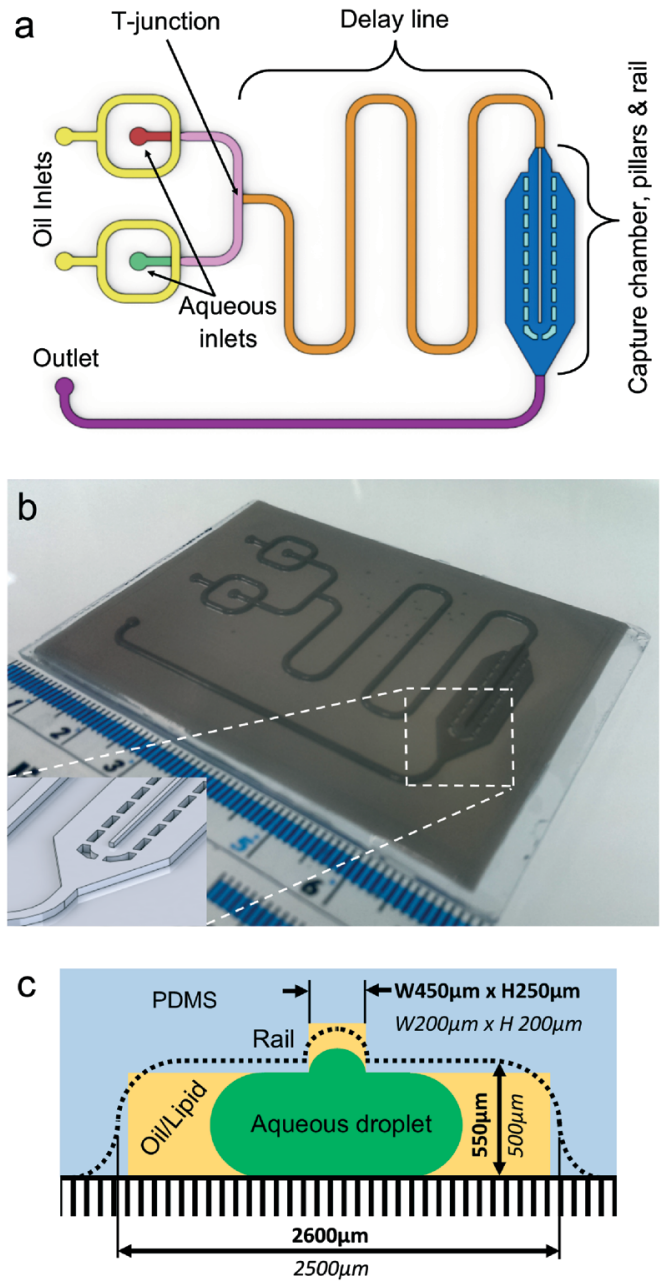

Fig. 2 Design of millifluidic chip for the generation of microliter droplets-in-oil and the formation of droplet arrays with interdroplet lipid bilayers. The chip features a pair of flow-focusing droplet generators (oil inlets in yellow and aqueous inlets in red and green), a delay line for lipid monolayer formation at the water-oil interface (orange), and a droplet capture chamber (blue) (a). The channels are $1.10 \mathrm{~mm}$ wide and $0.55 \mathrm{~mm}$ deep and are cast in PDMS from a 3D-printed mould with outer dimensions of $50 \times 70 \mathrm{~mm}$ (b). The droplet capture chamber includes a rail for droplet positioning. Measured values (bold) and CAD design values (italics) of the main features are given, with the CAD design profile shown in yellow/green and the actual profile of the capture chamber indicated by the dotted line (c).

flow and a $30 \mathrm{~min}$ bake step at $80^{\circ} \mathrm{C}$. This silanization procedure renders the glass cover of the channels hydrophobic, which enables aqueous droplets to flow easily through the channels.

\section{Device operation}

Pump 11 Plus syringe pumps (Harvard Apparatus, MA, USA) with TLC 1000 series syringes (Hamilton Bonaduz, Switzerland) were used to deliver the oil-lipid phase and one or two aqueous phases to the chip. For the aqueous solutions, the flow was controlled by the syringe pumps alone, which were connected to the aqueous inlets of the chip with push-fit PFA 
tubing (see Fig. 2a). Off-chip solenoid valves (LFE series PFE 2-way Micro Inert Valves (The Lee Company, CT, USA)) were used to split the flow from the single oil-lipid syringe pump into two tubes, which each connected to the oil inlet of a droplet generator (Fig. 2a). The oil phase consisted of hexadecane (Sigma Aldrich) with $40 \mathrm{mg} \mathrm{mL}^{-1}$ asolectin, a soybean lipid extract with $\approx 20 \%$ phosphatidylcholine lipids (Avanti Polar Lipids, AL, USA). The oil flow was set to 20-200 $\mu \mathrm{L} \min ^{-1}$ for droplet array formation, but was increased to $500 \mu \mathrm{L} \mathrm{min}{ }^{-1}$ to force the arrayed droplets out of the capture chamber, enabling new droplets to enter the pillar trap for the next experiment.

The microdroplet arrays were monitored using a Prosilica GX3300C digital camera (Allied Vision Technologies, Germany) with an $f=58 \mathrm{~mm}$ lens set to $\approx 0.25 \times$ magnification, giving a field of view of $\approx 70 \times 50 \mathrm{~mm}$. In the case of the melittin observations (Fig. 7) a Prosilica GC2450C was mounted on a Stereozoom Discovery.V8 microscope (Carl Zeiss, Germany) set at $1 \times$ zoom, with the image focused through a Zeiss PlanApoS $0.63 \times$ FWD $81 \mathrm{~mm}$ lens resulting in a $\approx 30 \times 25 \mathrm{~mm}$ field of view. Images were captured in time-lapse mode using custom software, before being processed and compiled into video format using ImageMagick ${ }^{25}$ and libav. ${ }^{26}$ For the still images in Fig. 7, local contrast enhancement followed by wavelet denoising was applied. ${ }^{27}$ The high resolution of the digital cameras allowed the subsequent cropping of the video images to highlight areas of interest without loss of quality.

\section{Electrical measurements}

A $5 \%(\mathrm{w} / \mathrm{v})$ solution of agar in $1 \mathrm{M} \mathrm{KCl}$ was prepared by stirring the initial agar dispersion at $120{ }^{\circ} \mathrm{C}$. Two plastic pipette tips were filled with the molten agar and a $\mathrm{Ag} / \mathrm{AgCl}$ wire electrode was inserted into each tip. The solidified agar gel formed a salt bridge from the electrode to the open ends of the pipette tips, which were inserted into the droplet capture chamber through the holes punched into the PDMS layer. The electrodes were connected to an ID562 bilayer amplifier (Industrial Developments Bangor, UK) to measure the capacitance of interdroplet lipid bilayers. A linear voltage ramp of $\pm 1 \mathrm{~V} \mathrm{~s}^{-1}$ was applied as a $50 \mathrm{mV}$ peak-to-peak triangle waveform of $500 \mathrm{~Hz}$ frequency and the capacitance was calculated from the measured current signal. ${ }^{28}$ For current measurements, the bilayer was voltage-clamped and the current signal was digitized with a sampling frequency of $5 \mathrm{kHz}$. All electrical measurements were performed without the use of a Faraday cage.

\section{Results and discussion}

\section{On-demand droplet generation}

To enable arraying of microliter droplets-in-oil with concomitant formation of large interdroplet lipid bilayers, the channels of our droplet device were $1100 \mu \mathrm{m}$ wide and $550 \mu \mathrm{m}$ deep, which is why we refer to a millifluidic rather than a microfluidic chip. The device, depicted in Fig. 2a, includes two flow-focusing droplet generators, supporting the use of two different aqueous phases, a delay line to allow time for lipid monolayer formation at the aqueous-oil interface, and a rail-guided and pillar-flanked droplet capture chamber. Hexadecane oil with $40 \mathrm{mg} \mathrm{mL} \mathrm{m}^{-1}$ asolectin lipids is continuously flowing through the chip.

To produce a droplet, the oil flow to the desired droplet generator is stopped by switching the external solenoid valve to the closed position. Next, the desired volume of an aqueous phase is introduced in the droplet generator at a flow rate of $10-20 \mu \mathrm{L} \mathrm{min} \mathrm{m}^{-1}$ by one of the two aqueous-phase syringe pumps. The oil flow to the droplet generator is then restored, which causes the aqueous volume in the channel beyond the oil-aqueous junction to be pinched off, forming an aqueous droplet in the oil stream. This on-demand droplet production method is based on Churski et al., who used solenoid valves on the aqueous channel. ${ }^{29}$ Our droplet generators can produces droplets with a volume between 0.7 and $6.0 \mu \mathrm{L}$. The lower volume limit is determined by the internal volume of the flow-focusing devices, whereas the upper limit is determined by the volume of the channel connecting the flow-focusing elements to the downstream elements of the chip (Fig. 2a, pink). If the aqueous slug is advance beyond this point, it meets the oil phase flowing through the other side of the chip, and is broken up into smaller slugs at the T-junction leading to the delay line.

The oil-lipid phase always flows through one of the two droplet generators, ensuring a constant flow of bulk oil phase through the delay line and the droplet capture chamber. The inclusion of the delay line improves droplet stability, i.e. reduces the tendency of contacting droplets to coalesce, by allowing sufficient time for the lipids to assemble as a monolayer at the droplet-oil interface. ${ }^{21,30}$ To ensure that the droplets pack in a linear array in the capture chamber while avoiding droplet merging, the device was operated with an oil flow rate of $20-200 \mu \mathrm{L} \mathrm{min}{ }^{-1}$.

\section{Droplet array formation}

At the point where the lipid-coated droplets exit the delay line and move into the capture chamber, the channel widens to allow for a continuous oil flow in the presence of stationary droplets. As subsequent droplets fill up the pillar-flanked droplet trap, the inter-droplet oil is expelled through the slits in-between the pillars, as illustrated in Fig. 3a. The minimum oil flow rate that results in sufficient pressure to ensure constant droplet-droplet contact is $20 \mu \mathrm{L} \mathrm{min}{ }^{-1}$. Higher oil flow rates enable tighter droplet packing, which results in larger interdroplet bilayers. For example, the images shown in Fig. 3b-c indicate that the interdroplet bilayers are $\approx 1350 \mu \mathrm{m}$ in width at an oil flow rate of $20 \mu \mathrm{L} \mathrm{min} \operatorname{mon}^{-1}$ and $\approx 2150 \mu \mathrm{m}$ in

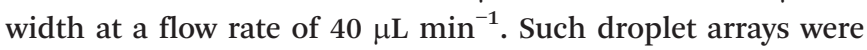
stable for 6 hours, at which point the experiments were always terminated because the oil syringe required refilling.

The time required to form a droplet array primarily depends on the time that it takes for the droplets to move down the capture chamber. We observed that it is crucial that 

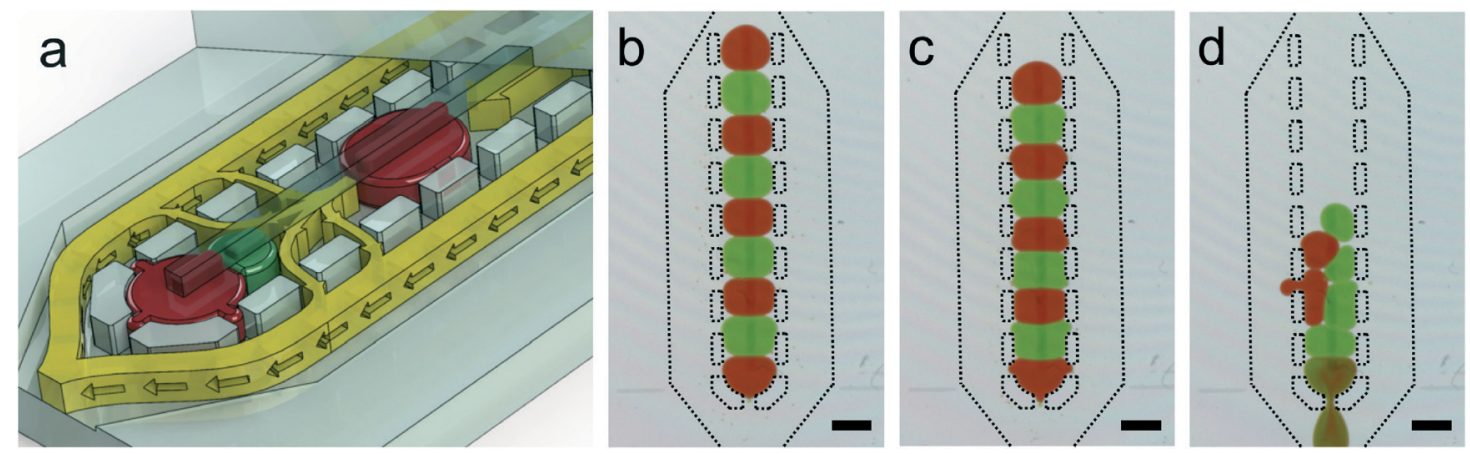

Fig. 3 Overview and demonstration of the array formation and reset processes. Droplets move into the pillar trap array because they follow the path of the continuous oil flow. As subsequent droplets become trapped in the pillar array, the interdroplet oil flows out through the slits between the pillars, the droplets come into direct contact, and an interdroplet lipid bilayer is formed (a). Arrays can be constructed with two types of droplets. An alternating array of $2 \mu \mathrm{L}$ droplets is shown, with the (transparent) device features overlaid on the video image. The array was formed at an oil flow rate of $20 \mu \mathrm{L} \mathrm{min}^{-1}$ (40 mg mL${ }^{-1}$ asolectin in hexadecane), with droplets containing green or red food dye, 100-fold diluted in buffer solution $\left(500 \mathrm{mM} \mathrm{KCl}, 10 \mathrm{mM} \mathrm{HEPES}, \mathrm{pH}\right.$ 7.5) (b). Increasing the flow rate to $40 \mu \mathrm{L} \mathrm{min}{ }^{-1}$ causes the droplets to pack more tightly, leading to larger interdroplet bilayers (c). The pillar trap can be emptied by raising the flow rate to $500 \mu \mathrm{L} \mathrm{min}^{-1}$, which causes the droplets to be pushed through the slits in-between the flanking pillars (d). Scale bar $=2 \mathrm{~mm}$. Array formation is shown in ESI. $\dagger$

a droplet reaches its final position within the array before it is contacted by the next droplet, as droplets that touch while both droplets are moving through the pillar trap tend to fuse. At an oil flow rate of $30 \mu \mathrm{L} \mathrm{min}{ }^{-1}$ a droplet that emerges from the delay line requires about 1 minute to reach the deepest position within the pillar trap, hence the assembly of a linear array of 9 droplets with alternating compositions, as shown in Fig. 3, can be achieved in just 5-6 minutes from the generation of the first droplet. This is considerably faster than droplet array formation by manual pipetting of microliter droplets into a bulk oil phase because lipid monolayer formation under oil-lipid flow conditions is more efficient than for a static oil phase. ${ }^{21,28}$ Higher oil flow rates, up to $\approx 200 \mu \mathrm{L} \mathrm{min}{ }^{-1}$, reduce array formation time but result in a higher pressure on the droplets.

At flow rates of $\approx 300 \mu \mathrm{L} \mathrm{min}^{-1}$ the droplets start to occupy the slits between flanking pillars of the droplet trap and small satellite droplets can split off from the original droplets. Conveniently, at a flow rate of $\approx 500 \mu \mathrm{L} \mathrm{min}^{-1}$ the increased force results in droplet ejection from the trap. This effect, shown in Fig. 3d, can be used to empty the droplet pillar trap, enabling formation of a new droplet array. It should be noted that these oil flow regimes depend on the viscosity of the oil phase, with lower-viscosity oils such as decane allowing higher flow rates than the hexadecane oil which was used in the present study.

\section{Variable-volume and non-linear droplet arrays}

The capture chamber has a rail running along its central axis, as depicted in Fig. 2c. When a droplet protrudes into this trench, its surface energy is reduced, causing the droplet to remain in contact with the trench in the absence of significant lateral forces. ${ }^{24}$ The trench in the roof of the PDMS capture chamber thus acts as a rail that guides the droplets along the centre of the pillar trap. The importance of this for the positioning of smaller droplets is illustrated in Fig. 4, which shows a droplet trap with three $3 \mu \mathrm{L}$ droplets and three $0.7 \mu \mathrm{L}$ droplets. Initially, the smaller droplets are positioned exactly in the middle of the pillar array because they adhere to the central rail. Hence, the droplet trap supports linear arrays of droplets that are smaller than the trap is wide. However, when the force acting on the smaller droplets is increased, they are displaced from the rail and form a 1.5D droplet packing pattern. In the experiment shown in Fig. 4 this is achieved by addition of the last $3 \mu \mathrm{L}$ droplet, but rail displacement can also be induced by switching to a higher oil flow rate.

\section{Interdroplet bilayers}

In ion channel electrophysiology, the formation of aperturesuspended bilayers or interdroplet bilayers is routinely

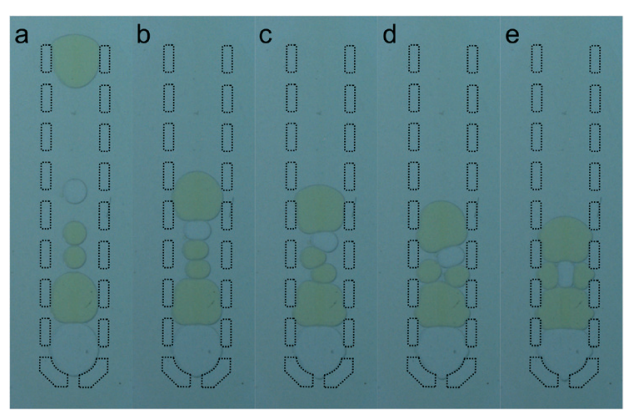

Fig. 4 Formation of more complex droplet networks. Small $(0.7 \mu \mathrm{L})$ droplets remain in the centre of the droplet trap $(a, b)$ until application of a threshold force (here an incoming larger droplet) displaces the droplets from the central rail (c, d). The result is a 1.5D packing pattern, where the third small droplet always occupies the centre position (e). Transparent droplets contain buffer solution $(500 \mathrm{mM} \mathrm{KCl}, 10 \mathrm{mM}$ HEPES, pH 7.5) whereas the yellow droplets also contain $250 \mu \mathrm{M}$ fluorescein dye. Array formation is shown in ESI. + 
assessed by capacitance measurements. ${ }^{31,32}$ The specific capacitance of a solvent-free lipid bilayer is $\approx 0.5 \mu \mathrm{F} \mathrm{cm}{ }^{-2,2,33}$ e.g. a circular bilayer of diameter $150 \mu \mathrm{m}$ should give a capacitance reading of $\approx 90 \mathrm{pF}$. A significantly smaller capacitance $(<10 \mathrm{pF})$ indicates that an oil film is present that has not (yet) thinned into a bilayer. To perform measurements on an interdroplet lipid bilayer in the droplet trap, two $\mathrm{Ag} / \mathrm{AgCl}$ electrodes were inserted through the roof of the capture chamber (Fig. 5, inset). The first droplet was introduced at an oil flow rate of $200 \mu \mathrm{L} \mathrm{min}{ }^{-1}$, passed the first electrode and remained in position under the second electrode. The second droplet was then introduced at the same flow rate, made contact with the first electrode and touched the already introduced droplet. At this point the flow rate was reduced to $20 \mu \mathrm{L} \mathrm{min}{ }^{-1}$ and the capacitance was recorded from the point at which a capacitance waveform became apparent. The time between the two droplets being formed was $50 \mathrm{~s}$, with the droplet pair being formed 2 minutes after the first droplet was produced. Fig. 5 shows for a particular experiment that the capacitance is $\approx 75 \mathrm{pF}$ for the first 3 minutes, then rapidly increases to $\approx 1.5 \mathrm{nF}$, which is followed by a gradual increase to $\approx 2.7-2.8 \mathrm{nF}$, which remains approximately constant after $\approx 25 \mathrm{~min}$. The initial capacitance of $\approx 75 \mathrm{pF}$ was attributed to a large-area oil film and the subsequent rapid increase in capacitance to the thinning of this film into a lipid bilayer with an area of $\approx 0.28 \mathrm{~mm}^{2}$. The bilayer then gradually increases in size, most likely due to a small but constant compression force from the oil flow, to an area of $\approx 0.56 \mathrm{~mm}^{2}$. A similar evolution of the capacitance is shown for three more experiments in the ESI. $\dagger$ The width of the pillar trap is $2.6 \mathrm{~mm}$, implying that a perfectly circular lipid bilayer would occupy $40 \%$ of the available space. Hence lipid bilayers with large areas are indeed formed between droplets that are

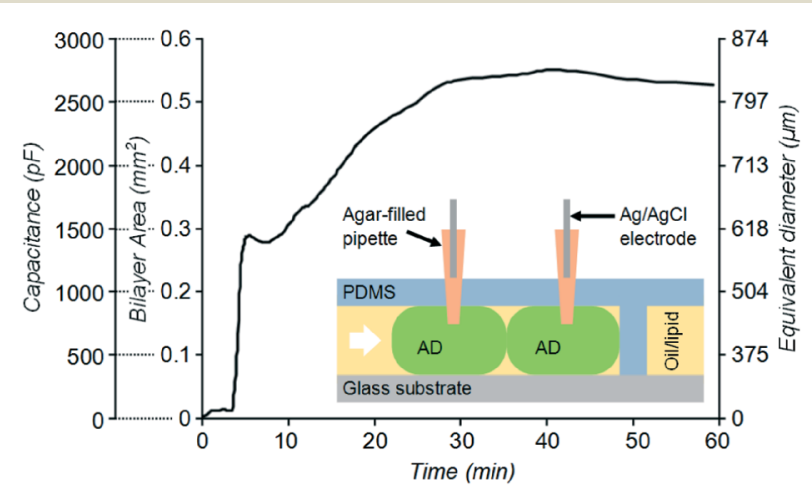

Fig. 5 Capacitance measurements of interdroplet bilayers. A pair of access holes are punched through the PDMS roof of the capture chamber to accommodate plastic pipette tips with $\mathrm{Ag} / \mathrm{AgCl}$ electrodes in an agar salt bridge (inset). The lipid bilayer capacitance was monitored over time, and was found to peak $\approx 30-40$ minutes after initial droplet contact at 2.5-2.8 nF. Assuming a bilayer capacitance of $0.5 \mu \mathrm{F} \mathrm{cm}$, this corresponds to an area of up to $0.56 \mathrm{~mm}^{2}$, and represents approximately $40 \%$ of the total available area in the pillar droplet trap. For comparison, a circular bilayer with the same area would have a diameter of up to $850 \mu \mathrm{m}$. $A D=$ aqueous droplets. Further capacitance data is shown in the ESI.† packed in the pillar array trap. We anticipate that bilayer formation takes place faster at higher flow rates, and that the final bilayer size can be tuned by adjusting the droplet volume and the oil flow rate.

Bilayer current measurements aimed at the study of single ion channels incorporated in interdroplet bilayers require isolation from electromagnetic, vibrational and acoustic noise sources, which we did not implement in the present study. However, as proof of principle we contacted a droplet of $1 \mathrm{M} \mathrm{KCl}$ and $10 \mathrm{mM}$ HEPES (pH 7.0) with a second droplet that also contained $10 \mu \mathrm{g} \mathrm{mL}{ }^{-1}$ of the nanopore $\alpha$-hemolysin, a water-soluble toxin from $S$. aureus, in our two-electrode droplet trap and recorded the bilayer current at a potential of $+50 \mathrm{mV}$. After applying a $20 \mathrm{~Hz}$ low-pass filter to reduce the peak-to-peak noise of the baseline signal, several current steps of $\approx 39 \mathrm{pA}$ were clearly visible, followed by a rapid increase in bilayer current and subsequent bilayer failure (see ESI $\dagger$ ). The discrete current steps, equivalent to a conductance of $\approx 780 \mathrm{pS}$, are in agreement with literature values for the $\alpha$-hemolysin conductance in $1 \mathrm{M} \mathrm{KCl}^{34}$ and the rapid increase in bilayer current is expected for sequential insertion of $\alpha$-hemolysin nanopores, which will destabilize the bilayer. Our observation of single-nanopore current events demonstrates the potential for single-channel electrical recordings with millifluidic interdroplet bilayers. It should be noted, however, that most ion channels will require implementation of proper shielding because gating typically occurs at a millisecond timescale and open amplitudes are relatively small, which precludes extensive filtering of the current signal.

\section{Bilayer permeation and perturbation}

The permeability of lipid bilayers, with or without incorporated ion channels or nanopores, can be optically assessed by monitoring colour changes in the droplets when the bilayertraversing species are coloured or fluorescent or when these initiate a (bio)chemical reaction in the receiving droplet that gives rise to a colour change. ${ }^{35,36}$ In the case of water transport over the bilayer, ${ }^{37}$ permeability can also be assessed by monitoring a change in size of the droplets on either side of the interdroplet bilayer. To illustrate the latter application, we created an array of three droplets, with a centre droplet of $4 \mu \mathrm{L}$ volume containing $0.25 \mathrm{M} \mathrm{KCl}$ and two flanking $2 \mu \mathrm{L}$ droplets with $1 \mathrm{M} \mathrm{KCl}$. As demonstrated in Fig. 6, the low-salt droplet decreases in volume over time, whereas the high-salt droplets increase in volume, indicative of osmotic water transfer from the low-salt to the high-salt droplets. ${ }^{38,39}$ From Fig. 6 we estimate that the high-salt droplet increased in volume by $2 \mu \mathrm{L}$ in a one-hour period. Assuming a total bilayer area of $1.0 \mathrm{~mm}^{2}$ (Fig. 5) between the low-salt and the two high-salt droplets, the osmotic permeability of the bilayer calculated according to Dixit et al. is $23 \mu \mathrm{m} \mathrm{s} \mathrm{s}^{-1}$, in good agreement with the literature. ${ }^{38}$ It should also be noted that the interdroplet bilayer array was stable despite the imposed osmotic gradient and that the dye molecules used to colour the droplets $(500 \mu \mathrm{M}$ Direct Red 23 and $250 \mu \mathrm{M}$ erioglaucine 


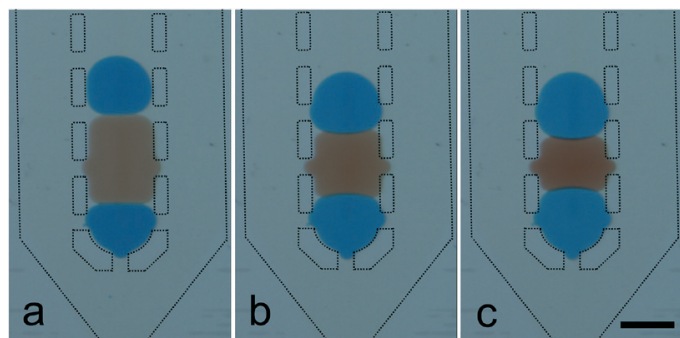

Fig. 6 Transfer of water over interdroplet bilayers. A $4 \mu \mathrm{L}$ redcoloured droplet of $0.25 \mathrm{M} \mathrm{KCl}$ solution is contacted by two $2 \mu \mathrm{L}$ blue-coloured droplets of $1 \mathrm{M} \mathrm{KCl}$ solution with a continuous oil-lipid flow of $30 \mu \mathrm{L} \mathrm{min}{ }^{-1}$. Over time ( $a=0 \mathrm{~min}, \mathrm{~b}=30 \mathrm{~min}, \mathrm{c}=1$ hour) the volume of the low-salt droplet decreased and the volume of the high-salt droplets increased, indicating osmosis driven transport of water over the interdroplet bilayers. All droplets contained $10 \mathrm{mM}$ HEPES $\mathrm{pH}$ 7.5. The scale bar is $2 \mathrm{~mm}$. The ESI $\uparrow$ shows array formation and osmotic transfer.

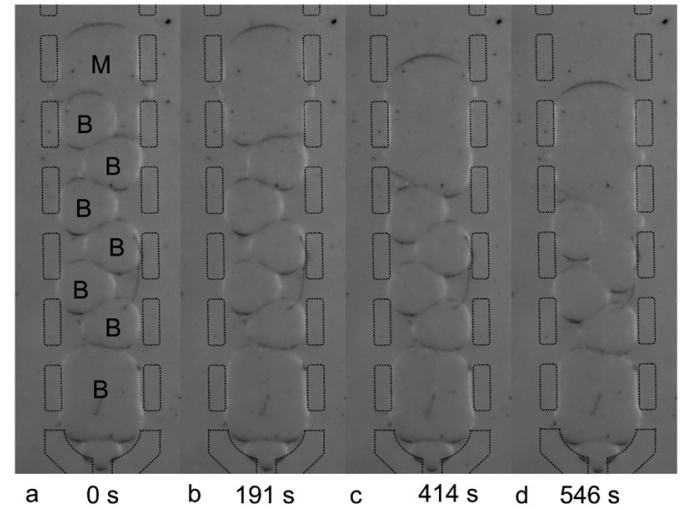

Fig. 7 Interaction of melittin with interdroplet bilayers. The pillar array is filled with one $3 \mu \mathrm{L}$ buffer droplet, 6 droplets of $1 \mu \mathrm{L}$ buffer, and with a $3 \mu \mathrm{L}$ droplet that contains $5 \mu \mathrm{g} \mathrm{mL} \mathrm{L}^{-1}$ melittin in buffer solution (a). Melittin causes the first interdroplet bilayer to fail, resulting in fusion of the original melittin droplet and the first buffer droplet (b). The next interdroplet bilayers in the array sequentially merge with the melittincontaining droplet, the melittin concentration of which is diluted with each fusion event $(c, d)$. The buffer solution is $10 \mathrm{mM} \mathrm{HEPES}, 200 \mathrm{mM} \mathrm{KCl}$, $\mathrm{pH}$ 7.5. The formation of the array and the subsequent effect of melittin are shown in the ESI.†

as the red and the blue dye, respectively) were not able to traverse the lipid bilayer.

The activity of biomolecules that cause lysis of biological cell membranes can be investigated by monitoring bilayer failure, which is readily visualized as merging of two adjacent droplets. As an example, we investigated the effect of the peptide melittin from bee venom, ${ }^{40}$ which at micromolar concentrations forms large pores in lipid bilayers, eventually leading to membrane disintegration in a detergent-like fashion. ${ }^{41} \mathrm{We}$ contacted a $3 \mu \mathrm{L}$ droplet containing $5 \mu \mathrm{g} \mathrm{mL}^{-1}(\approx 2 \mu \mathrm{M})$ melittin with a linear array of densely packed $1 \mu \mathrm{L}$ droplets of buffer solution (Fig. 7a). As can be seen by comparing Fig. $7 \mathrm{a}$ and $\mathrm{b}$, the melittin droplet fused with the adjacent buffer droplet, indicating failure of a melittin-destabilized interdroplet bilayer. The next interdroplet bilayer in the array was now in contact with the melittin solution (Fig. 7b) and also disintegrated (Fig. 7c), resulting in an increase in size of the melittin-containing droplet. Subsequent interdroplet bilayers in the array also failed when they became exposed to the melittin solution, but the rate of droplet fusion slowed down, which can be attributed to the gradual dilution of the original melittin solution with each droplet fusion event, leading to a reduced rate of pore formation.

\section{Conclusions}

We have developed a millifluidic device that allows the production of large-volume droplets and their controlled arrangement into stable linear droplet arrays, as well as more complex networks of droplets of variable volume. Our approach combines the automation of microfluidic devices with the electrical and optical accessibility of manually positioned microliter droplets. The large droplet volume enabled capacitance measurements of the interdroplet bilayers by insertion of electrodes and visualization of the volumemodulating effect of osmotic water transfer and the bilayerperturbing action of the toxin melittin with a CCD camera without magnifying optics.

It is noteworthy that microfluidic techniques developed for pico-to-nanoliter droplets (i.e. flow-focusing, droplets-onrails and pillar-flanked droplet traps) can be applied to microliter droplet generation and positioning by simply scaling the channel, rail and pillar size. We have demonstrated the potential of 3D printing for the manufacture of soft lithography moulds in this feature size regime, and realized a designto-device cycle of $<48$ hours by avoiding photolithography.

In summary, our millifluidic device allows automated and reproducible formation of stable linear droplet arrays with interdroplet bilayers for the study of membrane-active compounds, while the developed 3D printing approach enables rapid prototyping of additional droplet generators and/or alternative channel geometries, including branched pillar traps for droplet arrays with specific network properties. This technique simplifies the study of functional droplet networks, both in physiological and in technological contexts.

\section{Acknowledgements}

This research is supported in part by the European Commission through FET BioChemIT grant FP7-ICT-248992 "NeuNeu".

\section{Notes and references}

1 A. Huebner, S. Sharma, M. Srisa-Art, F. Hollfelder, J. B. Edel and A. J. de Mello, Lab Chip, 2008, 8, 1244-1254.

2 S. Y. Teh, R. Lin, L. H. Hung and A. P. Lee, Lab Chip, 2008, 8, 198-220.

3 A. B. Theberge, F. Courtois, Y. Schaerli, M. Fischlechner, C. Abell, F. Hollfelder and W. T. S. Huck, Angew. Chem., Int. Ed., 2010, 49, 5846-5868. 
4 H. Bayley, B. Cronin, A. Heron, M. A. Holden, W. L. Hwang, R. Syeda, J. Thompson and M. Wallace, Mol. BioSyst., 2008, 4, 1141-1224.

5 K. Funakoshi, H. Suzuki and S. Takeuchi, Anal. Chem., 2006, 78, 8169-8174.

6 R. Syeda, M. A. Holden, W. L. Hwang and H. Bayley, J. Am. Chem. Soc., 2008, 130, 15543-15548.

7 G. Maglia, A. J. Heron, W. L. Hwang, M. A. Holden, E. Mikhailova, Q. Li, S. Cheley and H. Bayley, Nat. Nanotechnol., 2009, 4, 437-440.

8 S. Leptihn, O. K. Castell, B. Cronin, E. H. Lee, L. C. M. Gross, D. P. Marshall, J. R. Thompson, M. Holden and M. I. Wallace, Nat. Protoc., 2013, 8, 1048-1057.

9 C. E. Stanley, K. S. Elvira, X. Z. Niu, A. D. Gee, O. Ces, J. B. Edel and A. J. de Mello, Chem. Commun., 2010, 46, 1620-1622.

10 Y. Bai, X. He, D. Liu, S. N. Patil, D. Bratton, A. Huebner, F. Hollfelder, C. Abell and W. T. S. Huck, Lab Chip, 2010, 10, 1281-1285.

11 Y. Elani, A. J. deMello, X. Niu and O. Ces, Lab Chip, 2012, 12, 3514-3520.

12 S. Aghdaei, M. E. Sandison, M. Zagnoni, N. G. Green and H. Morgan, Lab Chip, 2008, 8, 1617-1620.

13 M. R. R. de Planque, S. Aghdaei, T. Roose and H. Morgan, ACS Nano, 2011, 5, 3599-3606.

14 L. Yi-Ying, C. Ching-Wen, C. Li-Chi and F. Shih-Kang, Proceedings of the IEEE International Conference on Nano/Micro Engineered and Molecular Systems (NEMS), 2011, pp. 964-967.

15 M. S. Friddin, N. P. Smithers, M. Beaugrand, I. Marcotte, P. T. F. Williamson, H. Morgan and M. R. R. de Planque, Analyst, 2013, 138, 7294-7298.

16 A. Adamatzky, J. Holley, P. Dittrich, J. Gorecki, B. De Lacy Costello, K.-P. Zauner and L. Bull, BioSystems, 2012, 1, 72-77.

17 G. F. Christopher and S. L. Anna, J. Phys. D: Appl. Phys., 2007, 40, R319.

18 S. Thutupalli, S. Herminghaus and R. Seemann, Soft Matter, 2011, 7, 1312-1320.

19 M. Zagnoni and J. M. Cooper, Lab Chip, 2010, 10, 3069-3073.

20 T. Nisisako, S. A. Portonovo and J. J. Schmidt, Proceedings of the 16th International Conference on Miniaturized Systems for Chemistry and Life Sciences ( $\mu T A S), 2012$, pp. 88-90.
21 S. Thutupalli, J.-B. Fleury, A. Steinberger, S. Herminghaus and R. Seemann, Chem. Commun., 2013, 49, 1443-1445.

22 A. Bonyár, H. Sántha, B. Ring, M. Varga, J. Gábor Kovács and G. Harsányi, Procedia Eng., 2010, 5, 291-294.

23 M. C. Huang, H. Ye, Y. K. Kuan, M.-H. Li and J. Y. Ying, Lab Chip, 2009, 9, 276-285.

24 P. Abbyad, R. Dangla, A. Alexandrou and C. N. Baroud, Lab Chip, 2011, 11, 813-821.

25 ImageMagick, http://www.imagemagick.org, September 17, 2013.

26 libav, http://www.libav.org, September 17, 2013.

27 D. Tschumperle, 2013, G'MIC : Greyc's magic for image computing, http://gmic.sourceforge.net, November 11, 2013.

28 N. Malmstadt, M. A. Nash, R. F. Purnell and J. J. Schmidt, Nano Lett., 2006, 6, 1961-1965.

29 K. Churski, P. Korczyk and P. Garstecki, Lab Chip, 2010, 10, 816-818.

30 J. C. Baret, F. Kleinschmidt, A. El Harrak and A. D. Griffiths, Langmuir, 2009, 25, 6088-6093.

31 S. Demarche, K. Sugihara, T. Zambelli, L. Tiefenauer and J. Vörös, Analyst, 2011, 136, 1077-1089.

32 P. Kongsuphol, K. B. Fang and Z. Ding, Sens. Actuators, B, 2013, 185, 530-542.

33 Y. Cheng, R. J. Bushby, S. D. Evans, P. F. Knowles, R. E. Miles and S. D. Ogier, Langmuir, 2001, 17, 1240-1242.

34 G. Menestrina, M. Dalla Serra, M. Comai, M. Coraiola, G. Viero, S. Werner, D. A. Colin, H. Monteil and G. Prévost, FEBS Lett., 2003, 552, 54-60.

35 S. Li, P. C. Hu and N. Malmstadt, Biophys. J., 2011, 101, 700-708.

36 M. Dezi, A. Di Cicco, P. Bassereau and D. Lévy, Proc. Natl. Acad. Sci. U. S. A., 2013, 110, 7276-7281.

37 M. B. Lande, J. M. Donovan and M. L. Zeidel, J. Gen. Physiol., 1995, 106, 67-84.

38 S. S. Dixit, A. Pincus, B. Guo and G. W. Faris, Langmuir, 2012, 28, 7442-7451.

39 Z. Michalak, D. Fartash, N. Haque and S. Lee, CrystEngComm, 2012, 14, 7865-7868.

40 H. Raghuraman and A. Chattopadhyay, Biosci. Rep., 2007, 27, 189-223.

41 M. T. Lee, T. L. Sun, W. C. Hung and H. W. Huang, Proc. Natl. Acad. Sci. U. S. A., 2013, 110, 14243-14248. 